\title{
Greater visual working memory capacity for visually- matched stimuli when they are recognized as meaningful
}

\author{
Isabel E. Asp ${ }^{1,2 *}$, Viola S. Störmer ${ }^{1,3}$, Timothy F. Brady ${ }^{1}$ \\ ${ }^{1}$ Department of Psychology, University of California, San Diego, \\ La Jolla, California, 92093 \\ ${ }^{2}$ Veterans Affairs San Diego Healthcare System, \\ La Jolla, California, 92161 \\ ${ }^{3}$ Neuroscience Graduate Program, University of California, San Diego, \\ La Jolla, California, 92093
}

Please address correspondence to:

Timothy Brady

timbrady@ucsd.edu

Department of Psychology, University of California, San Diego 9500 Gilman Dr. \#0109, La Jolla, CA 92093 


\begin{abstract}
Almost all models of visual working memory assume it has a fixed capacity: some models propose a limit of 3-4 objects, where others propose there is a fixed pool of resources for each basic visual feature. Recent findings, however, suggest that memory performance is improved for real-world objects. What supports these increases in capacity? Here, we test whether the meaningfulness of a stimulus alone influences working memory capacity while controlling for visual complexity and directly assessing the active component of working memory using EEG. Participants remembered ambiguous stimuli that could either be perceived as a face or as meaningless shapes. Participants had higher performance and increased neural delay activity when the memory display consisted of more meaningful stimuli and when they subjectively perceived the stimuli as meaningful. Thus, there are genuine increases in working memory capacity that arise from the subjective perception of the stimulus as meaningful.
\end{abstract}

Keywords: Visual working memory; working memory capacity; contralateral delay activity; EEG; meaningfulness; perceptual organization

\title{
Significance
}

Visual working memory is the system that holds visual information in an active state, making it available for cognitive processing and protecting it against interference. The capacity of this memory system is widely studied because it is related to academic achievement, fluid intelligence, and other important cognitive functions. Here, we demonstrate that identical visual objects recruit different amounts of working memory resources - as measured by both behavioral measures of capacity and neural measures of active maintenance - when they are recognized as meaningful than when they are not. At the broadest level, these data suggest working memory is not fixed capacity; instead, its capacity is dependent on exactly what is being remembered, with more meaningful stimuli recruiting additional memory resources. This suggests that widely used measures of working memory capacity that solely focus on meaningless objects are not accurate descriptions of individuals' capacity or of the working memory system. 


\section{EFFECT OF STIMULUS MEANING ON VWM}

\section{Introduction}

Visual working memory is a cognitive system used to hold visual information in an active state, protecting it from interference from new sensory inputs. The capacity of visual working memory is clearly highly limited. However, nearly all theories of visual working memory go beyond arguing capacity is limited and assert a 'fixed' capacity (e.g., of 3-4 objects; Awh, Barton \& Vogel, 2007; Luck \& Vogel, 1997; or a fixed resource pool: Bays et al. 2009). This fixed capacity is claimed to arise from limits in attentional capacity like a fixed number of attentional spotlights for maintaining items in the focus of attention (e.g., Cowan, 2005); or from more neurally-inspired resource limits like a fixed number of action potentials for each basic feature due to divisive normalization (e.g., Ma, Husain \& Bays, 2014; Bays, 2014), with the idea that visual working memory is fundamentally based on storage of basic features in low-level sensory cortex (e.g., Serences, 2016).

Contrary to this view of visual working memory as consisting of fixed-pools of resources for basic features (color, orientation, etc.), or fixed numbers of objects, some behavioral work has shown that familiarity and knowledge improve performance in short-term memory tasks, even for physically similar stimuli (e.g., Alvarez \& Cavanagh, 2004; Jackson \& Raymond, 2008; Brady et al. 2009; Curby et al. 2009; Ngiam et al. 2017). However, such results could potentially arise from the recruitment of more stable 'long'-term memory systems rather than changes in active storage in working memory (as claimed by, e.g., Lin \& Luck, 2012; Quirk, Adam \& Vogel, 2020). To test for changes in working memory capacity per se, it is necessary to directly measure 'online', active storage of the items. One recent study aimed to do this, finding not only higher working memory performance but also increased neural delay activity for familiar realworld objects relative to simple features (Brady, Störmer \& Alvarez, 2016; c.f. Quirk et al. 2020). However, this study compared extremely disparate stimuli: real-world objects differ from simple features like colors not only in that they connect to existing knowledge (i.e., are meaningful), but they are also visually more complex, perhaps allowing the recruitment of additional sensory regions coding for different basic visual features. Thus, any differences observed behaviorally and particularly in neural markers may well be due to differences in visual information rather than meaningfulness per se. Thus, whether the meaningfulness of a stimulus alone can enhance active, online storage in working memory remains unknown, despite its critical importance to theories of the nature of visual working memory capacity.

To address this, here we use a novel test of the role of meaningfulness of a stimulus while nearly perfectly matching visual complexity across a series of experiments. We asked participants to remember two-tone images that were either perceived as meaningful or not for short periods of time, and measured memory performance (Exp.1 \& 2) as well as sustained neural activity during the maintenance period using electroencephalography as a direct measure of the active component of working memory (EEG; Exp. 3). Stimuli consisted of ambiguous face images ("Mooney images"; Mooney, 1957) that were carefully matched so that different images would be recognized as a face by different people (Brady, Alvarez, Störmer, 2019; Schwiedrzik et al. 2018); that is, the exact same image could be perceived as meaningful by one 


\section{EFFECT OF STIMULUS MEANING ON VWM}

person but as not meaningful by the next. Thus, among these face stimuli there were on average no physical differences between images that one participant would perceive as a face or perceive as a set of black-and-white arbitrary shapes (i.e., not meaningful). In addition to these ambiguous face stimuli we also showed participants arbitrary shapes that matched the overall low- and midlevel features of the faces (Exp. $1 \& 3$ ) or inverted versions of the faces (Exp. 2), which provided objective non-face conditions. This set of experiments allowed us to examine both the cognitive and neural consequences of visual information being perceived as meaningful and thus to examine whether active storage in working memory per se was changed by this recognition, independent of the physical attributes of the stimulus.

\section{Experiment 1: Behavioral capacity for Mooney faces vs. unrecognizable shapes Methods}

Participants

All participants gave written informed consent before beginning the experiment. Experiment 1 was preregistered (http://aspredicted.org/blind.php? $\mathrm{x}=\mathrm{k} 8 \mathrm{rm} 4 \mathrm{k}$ ) and, in accordance with the preregistration, 24 undergraduates from the University of California, San Diego participated for course credit (16 female, ages 18-28). All experiments were approved by the Institutional Review Board of the University of California San Diego.

\section{Stimuli}

The stimuli used consisted of 300 images (150 ambiguous Mooney faces and 150 shuffled Mooney faces that appeared as unrecognizable shapes). The ambiguous face images were previously ranked as moderately difficult to recognize a face, gaining the status "ambiguous" instead of "easy" (for details, see Schwiedrzik, Melloni, \& Schurger, 2018; Brady, Alvarez, \& Störmer, 2019). This difficulty was chosen in an attempt to create a more evenly distributed subjective report of faces seen. The unrecognizable shape stimuli were created by segmenting the Mooney face images into continuous regions of white or black, labeling these as 'parts'; inverting each region; and then creating new images from this library of parts, subject to the constraint that no 2 parts of a newly generated image overlapped (Fig 1B). The target/foil images for the 2-alternative forced-choice (2-AFC) test were randomly selected per trial; however, the foil image always matched the condition of the target (i.e., target: unrecognizable shapes; foil: unrecognizable shapes). To ensure that participants saw each stimulus an equal number of times and so that we could analyze item effects, each stimulus appeared 1 time as a target, 1 time as a foil, and twice as a distractor, giving a total of 300 trials per participant.

\section{Procedure}

During the experiment, participants viewed 3 images centered around fixation for 1000ms (Fig. 1A). We used long encoding times because of previous evidence that the role of meaningfulness may require long encoding times to become apparent (Brady et al. 2016). Such long encoding times also ensure we are tapping into visual working memory capacity, rather than encoding 


\section{EFFECT OF STIMULUS MEANING ON VWM}

limitations, as previous work has clearly shown that simple stimuli are encoded well within only a few hundred milliseconds (Luck \& Vogel, 1997; Vogel et al. 2006) and more complex stimuli, like the Mooney faces, within 500ms (Alvarez \& Cavanagh, 2004).

The memory display of each trial would consist of either $0,1,2$, or 3 ambiguous Mooney faces with unrecognizable shape stimuli making up any remainder so that the memory set would always consist of 3 images. As such, we varied how many of these images were ambiguous face stimuli vs. unrecognizable shape stimuli throughout the experiment. While the images were on the screen, participants were to maintain their eyes on the center of the screen. After the images disappeared, there was an $800 \mathrm{~ms}$ delay period with only image location markers on the screen. Following the delay, one of the location markers enlarged for 500ms, indicating the location of the to-be-tested image. The target image and a foil image of the same condition (i.e., face or unrecognizable shapes) were then presented one above the other, with the space between the two images centered at the prior location marker. Participants were asked to indicate which of the two images was part of the recent set of studied images by pressing a key on the keyboard (top image: upper arrow key; bottom image: lower arrow key). The two images remained on the screen until the participant made a selection. Once the participant pressed a key, they were prompted to recall and respond with how many images they had perceived as faces during encoding $(0,1,2$, or 3$)$ using the number pad. The experiment consisted of 5 blocks with 60 trials in each. The trial conditions were randomized throughout the experiment and each trial condition was presented evenly within each block. Prior to the experiment, all participants completed two mini-blocks of 4 trials each as practice for the full five blocks of the experiment that followed. None of the images used in the practice trials were used in the full experiment.

\section{Statistical Analyses}

For all experiments, our main analysis uses a Generalized Liner Mixed Effect model that treats both participants and items as random effects. This allows us to test whether memory effects were robust across our stimulus set. We fit two models: first, asking about the effect of objective number of faces shown (0-4); second, asking about the effect of both objective and subjective number of faces and their interaction. Details of this analysis can be found in the Supplementary Material.

We also assessed performance across the objectively-shown number of faces with a repeated-measures ANOVA, which does not take into account the effects of individual items but may be more interpretable for readers unfamiliar with the mixed effect approach. 

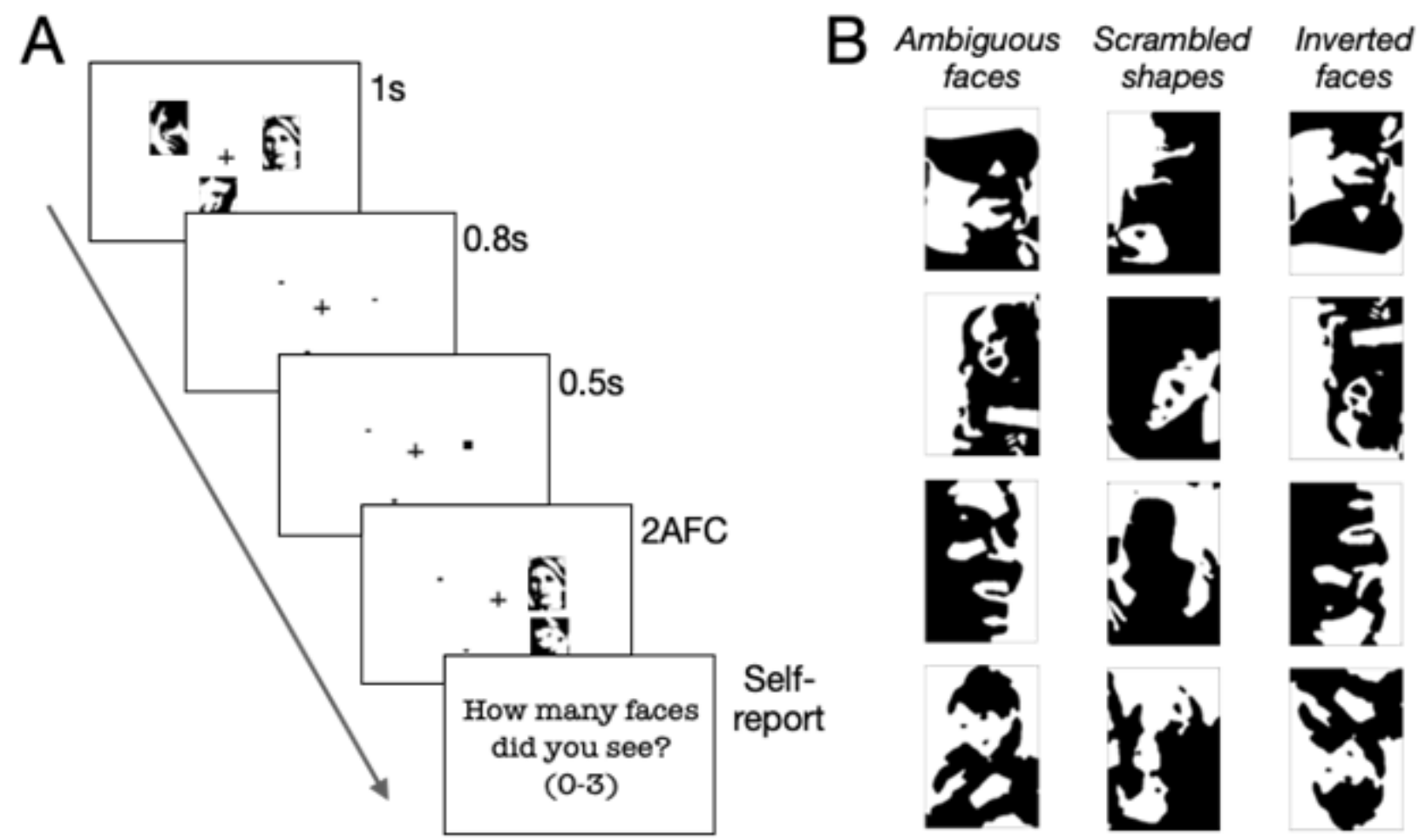

Figure 1. (A) Experiment 1 and 2 methods. Participants saw a memory display with three images that could either be ambiguous Mooney faces or non-faces. The non-faces in Experiment 1 were scrambled versions of the Mooney faces ('unrecognizable shapes') and in Experiment 2 were inverted Mooney faces. After a short delay, participants were shown two images and had to indicate which one was part of the memory display (top vs. bottom). Subsequently they were asked how many faces they perceived during encoding. (B) Example stimuli: ambiguous Mooney faces (used in all Experiments); Unrecognizable shape stimuli made from scrambling Mooney images (used in Exp. 1 and 3); Inverted ambiguous Mooney images (used in Exp. 2).

\section{Results}

Recognition accuracy increased when more faces, rather than unrecognizable shape images, were presented during the study phase $(F(3,69)=18.7, p<0.0001)$, despite the unrecognizable shapes being made from the same underlying set of shapes and correspondingly having the same lowand mid-level features present as the faces. A binomial general linear mixed effect model showed this finding was robust not only across individuals, but also across items (individual faces/unrecognizable shapes): $\beta=0.18, F(1,7198)=34.2, p<0.0001$.

We also analyzed the data as a function of both the objective number of faces present and the subjective number of faces participants reported perceiving and found a significant effect of number of subjective faces $(\beta=0.15, F(1,7196)=4.82, p=0.028)$, no significant effect of the objective number of faces $(\beta=-0.03, F(1,7196)=0.36, p=0.55)$, and an interaction between these two factors: $(\beta=0.09, F(1,7196)=6.82, p=0.009$; Fig. $2 \mathrm{~A}-\mathrm{B})$. Thus, while taken alone there was both an effect of the objective and subjective number of faces, the effect of subjective number of faces seen on accuracy was larger when objectively more faces were shown. Furthermore, 


\section{EFFECT OF STIMULUS MEANING ON VWM}

because items were included as random effects in the general linear mixed effect model we can conclude that the memory improvement was robust across the images. Figure $2 \mathrm{C}$, where we plotted individual performance (correct/incorrect) for each stimulus tested, shows that there was no consistency in which images were more likely to be remembered (Fig. 2C).
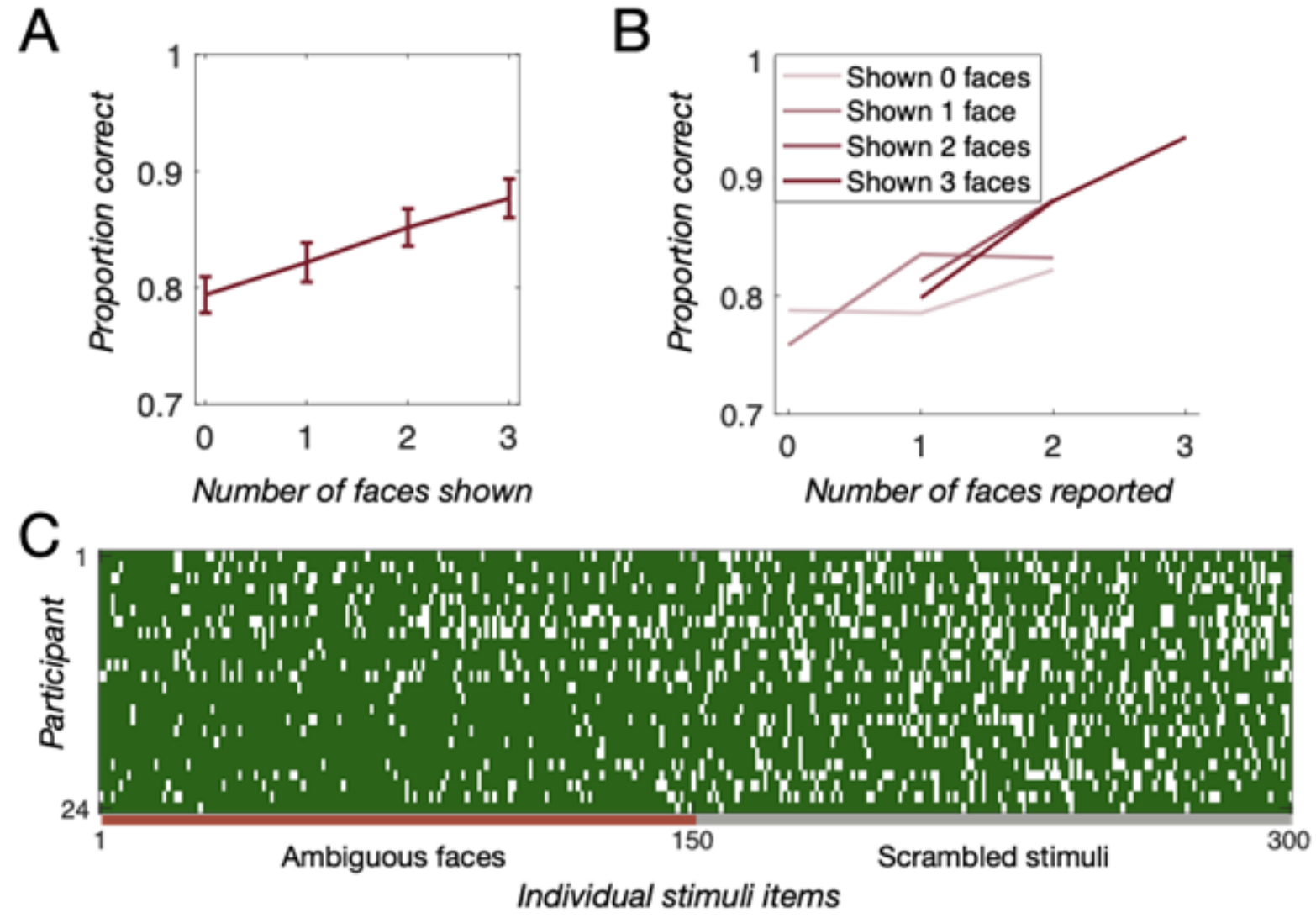

Figure 2. Results of Experiment 1. (A-B) Accuracy (proportion correct) for the 2-AFC memory match judgment depended on $(A)$ how many faces were shown in the memory display $(B)$ and how many faces participants reported seeing, such that more faces perceived tended to result in better performance when more faces were shown. (C) Individual trials, where green indicates a correct response and white indicates an incorrect response. The lack of vertical columns indicates that individual items did not drive the effect, as although participants remembered faces better than scrambled images, participants were not consistent in which faces or scrambled images tended to be accurately reported.

\section{Experiment 2: Behavioral capacity for Mooney faces vs. inverted faces}

In Experiment 2 we used inverted versions of the ambiguous Mooney images to more tightly control for image features between the recognizable and unrecognizable images since the exact same low- and mid-level features are present, although it also allows for the possibility that some inverted faces are recognized and/or that participants might mentally rotate the images some proportion of the time. Convergence between Experiment 1 and Experiment 2 would suggest that the effects we report are robust to how we choose to control for low- and mid-level features. 


\section{Methods}

\section{Participants}

Experiment 2 was preregistered (http://aspredicted.org/blind.php? $\mathrm{x}=\mathrm{tt} 4 \mathrm{pq} 2$ ) and, in accordance with the preregistration, 24 undergraduates from the University of California, San Diego participated, also for course credit (19 female, ages 18-32). One participant had performance below our preregistered exclusion criterion (average memory performance across conditions below chance) and was replaced.

\section{Stimuli and Procedure}

Experiment 2 was identical to Experiment 1 except that rather than using scrambled Mooney faces images, it used inverted Mooney faces (Fig 1B).

\section{Results}

Statistical analysis was identical to Experiment 1. Recognition accuracy increased when more faces, rather than inverted faces, were presented during the study phase $(F(3,69)=6.83$, $p=0.0004)$. This finding was robust not only across individuals, but also after taking into account the effect of items: $\beta=0.11, F(1,7198)=14.3, p=0.0002$.

When examining the effects of both the objectively-shown and subjectively-reported number of faces, we found no main effect of number of subjective faces $(\beta=0.03, F(1$, $7196)=0.23, p=0.63)$, no main effect of the objective number of faces $(\beta=-0.09, F(1,7196)=2.98$, $p=0.08)$, but an interaction $(\beta=0.11, F(1,7196)=13.1, p=0.0003$; Fig 3A-B). That is, just like in Experiment 1, there was an effect of the subjective number of faces reported that was larger when more faces were objectively present. In this case, the main effect of subjective faces was not significant, only the interaction, because this effect was more clearly modulated by the number of objective faces. In addition, looking at individual performance for each stimulus tested revealed no evidence of any reliable item effects which again suggests there was no consistency in which images were more likely to be remembered (Fig. 3C). 

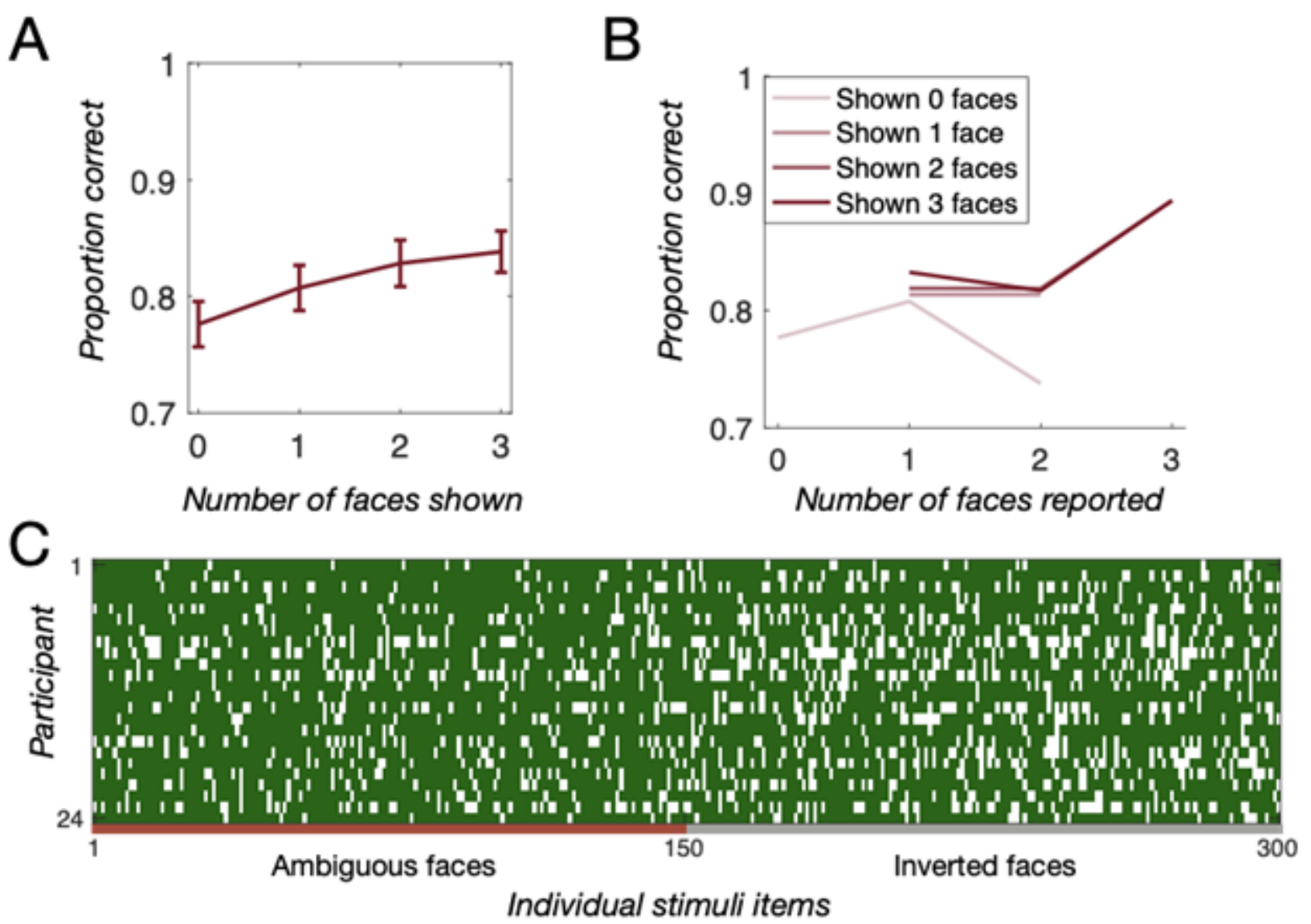

Figure 3. Results of Experiment 2. (A-B) Accuracy (proportion correct) for the 2-AFC memory match judgment depended on (A) how many faces were shown in the memory display $(B)$ and how many faces participants reported seeing, such that more faces perceived tended to result in better performance when more faces were present. (C) Individual trials, where green indicates a correct response and white indicates an incorrect response. The lack of vertical columns indicates that individual items did not drive the effect, as although participants remembered faces better than inverted-face images, participants were not consistent in which faces or inverted-face images tended to be accurately reported.

\section{Experiment 3: Active storage assessed via the contralateral delay activity}

The results of Experiments $1 \& 2$ provide initial evidence that visual working memory performance may benefit from the perceived meaningfulness of the stimulus. However, changes in behavioral performance alone do not provide conclusive evidence as to whether these stimuli are maintained actively in working memory, or whether episodic memory or activated forms of long-term memory are used - as long-term memory is known to be influenced by such meaning (Bartlett, 1932; Brady et al, 2019; Wiseman \& Niesser, 1974; Cowan, 2005). Thus, we next recorded the brain's electrophysiological responses that provide a direct measure of working memory engagement in Experiment 3 (CDA component; Vogel \& Machizawa, 2004). 


\section{Methods}

\section{Participants}

Twenty-three participants completed Experiment 3 and received course credit or $\$ 10$ per hour for participation. Data from three participants in Experiment 3 had to be excluded due to artifacts in the EEG (> 50\% of trials excluded), and data from one participant had to be excluded because part of the session was not recorded. Of the remaining 19 participants (13F, ages 18-34) all had normal or corrected-to-normal vision.

\section{Stimuli and Procedure}

We adapted the experiment to an EEG version of the task and showed the stimuli on a lateralized display with three images in each visual half-field, and cued participants with a central arrow to only memorize one side of the display (either left or right; Figure 4A). This allowed us to measure the contralateral-delay activity (CDA), a neural marker of active maintenance of visual information (Vogel \& Machizawa, 2004). Just like in Exp. 1, we presented ambiguous face stimuli and scrambled versions of them, but this experiment only included two conditions: 1 face (and two unrecognizable shape images) vs. 3 faces, to obtain a sufficient number of trials. Because there were now 6 stimuli being displayed in a single study screen, the number of times each image was repeated increased. Face images were repeated $\sim 8-9$ times and unrecognizable shape images were repeated about 4 times throughout the experiment. This was due to the trial conditions that were chosen (1-face and 3-face trials). Importantly, if the participant successfully attended to one side only, the high repetition would not be overtly noticeable, since only half of the number of repetitions were on the task-relevant side of the display. More details on the experimental design are reported in the Supplementary Material. 

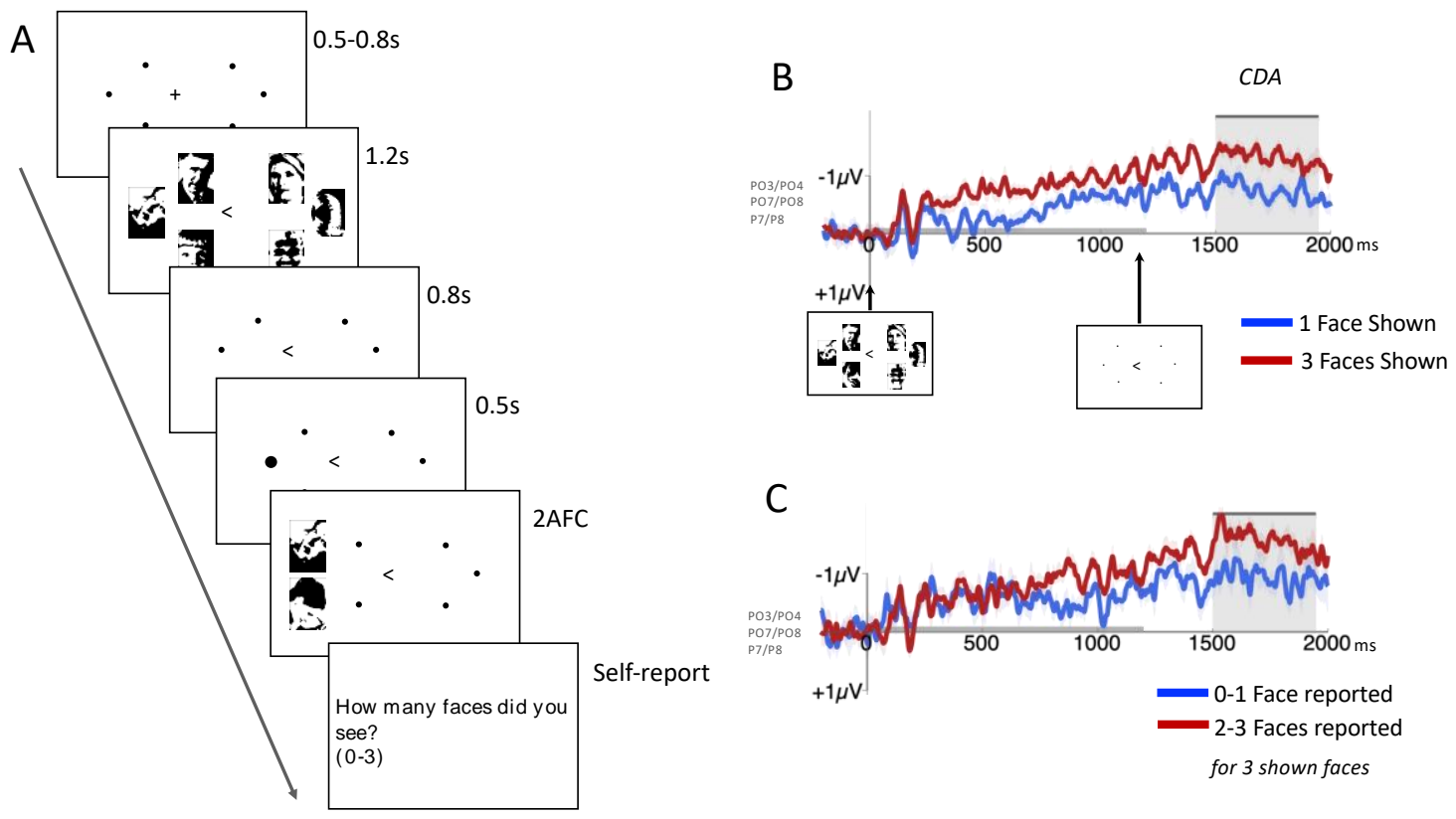

Figure 4. Task structure and results of Experiment 3. (A) Participants were cued to remember the items on one side of the display only (left or right). (B) Contralateral-minus-ipsilateral difference waveform over parietal-occipital electrode sites for when 1 face was shown (blue line) vs. 3 faces were shown (red line). (C) Contralateral-minus-ipsilateral difference waveform over parietal-occipital electrode sites for when 3 faces were shown, broken down by when 0-1 faces were reported (blue line) vs. 2-3 faces were reported (red line). The CDA is measured 300ms after offset of the memory display until the test probe appears (shaded gray area), and shaded areas represent within-subject standard errors of the mean at each time point.

Electrophysiological Recordings and Analysis

EEG activity was recorded continuously using our conventional recording and analysis procedures (Brady, Alvarez , \& Störmer, 2019; Keefe \& Störmer, 2012). Data were epoched into trials and artifact rejection was performed for the interval -200 to $+2000 \mathrm{~ms}$ relative to the memory display onset and trials with horizontal eye movements, blinks, or excessive muscle movements were excluded from the analysis. Artifact-free data was re-referenced to the average of the left and right mastoids, digitally low-passed filtered (half-amplitude cutoff at $30 \mathrm{~Hz}$; slope of $12 \mathrm{~dB}$ /octave), and baselined to the $200 \mathrm{~ms}$ pre-stimulus interval. Event-related potentials (ERPs) elicited by the memory display were averaged separately for each condition (1 face shown, 3 faces shown) and were then collapsed across to-be-remembered hemifield (left, right) and hemisphere of recording (left, right) to obtain waveforms recorded contralaterally and ipsilaterally to the to-be-remembered side. Finally, because the CDA is quantified as the difference in activity across hemispheres, we calculated contralateral-minus-ipsilateral difference 


\section{EFFECT OF STIMULUS MEANING ON VWM}

waveforms for each condition. For each participant, the mean CDA amplitude was measured with respect to the 200-ms pre-stimulus baseline at six posterior electrode sites

(PO3/PO4/PO7/PO8/P7/P8) beginning 300ms after the offset of the memory display until the test probe appeared (in this experiment, 1500-1950ms post memory display onset; cf. McCollough et al., 2007; Brady et al., 2016). The resulting mean amplitudes were our measure of the CDA magnitude. The exact electrode sites were chosen a priori based on a pilot subject that was not used in the main analysis. To ensure that the results were not due to the specific electrodes we used, we repeated all statistical analysis using electrode sites PO3/PO4/PO7/PO8 that match our previous work (Brady et al., 2016); all effects remain significant (main CDA effect: $p=0.028$; subjective report CDA effect: $p=0.042$ ). Details of EEG recording, checks for successful horizontal eye movement removal, and analysis procedures can be found in the Supplementary Material.

\section{Statistical Analyses}

The main analyses applied a Generalized Liner Mixed Effect model, just as in Exp. 1 \& 2, and thus used all $\sim 5000$ trials to estimate the effects of objective and subjective faces. Model details can be found in the supplement. However, for a follow-up subjective-report analysis, we also report the effect of looking only at performance with 3 objective faces present, which uses only a subset of the data used in the main mixed effect analysis. We averaged ERPs according to individual's reports of how many faces they reported seeing on each trial but only included those trials on which we showed three ambiguous faces. To have a sufficient number of trials in each condition, we collapsed across reports where participants said they saw 0 or 1 faces, and 2 or 3 faces. Furthermore, for this particular analysis, we a priori restricted our EEG analysis to those participants who had a minimum of 20 trials in each bin, resulting in 14 usable participants for this analysis. On average, the remaining participants had well over 20 trials per bin, with the average number of trials per bin being 67 in these participants.

\section{Results}

In line with Experiments 1 and 2, we found that recognition accuracy increased when more faces, rather than unrecognizable shapes, were presented during the study phase $(\mathrm{t}(18)=2.63$; $p=0.017)$ and this also held after taking into account the effect of items $(\beta=0.076, F(1$, 4964) $=4.1, p=0.04)$.

Importantly, this performance benefit was accompanied by increased CDA for the three vs. one-face condition $(t(18)=2.92 ; p=0.009$; Fig. $4 \mathrm{~B})$, which was also robust when taking into account the random effect of items $(\beta=0.035, F(1,7198)=6.12, p=0.01)$. Thus, there is a change not only in behavioral performance but this change is also reflected in increased CDA when more faces are shown, suggesting more information is actively held in visual working memory for faces than non-faces.

To test to what extent the increase in working memory capacity was due to subjectively perceiving the stimuli as faces, we again looked at behavioral performance as a function of 


\section{EFFECT OF STIMULUS MEANING ON VWM}

subjectively reported number of faces. We found both a main effect of number of subjective faces $(\beta=0.21, F(1,4962)=5.46, p=0.019)$, a main effect of the objective number of faces $(\beta$ $=0.16, F(1,4962)=4.35, p=0.037)$, and, in this case, no significant interaction $(\beta=-0.07, F(1$, $4962)=2.7, p=0.098$ ). The pattern of results was nearly identical to Exp. 1 and 2 , in that objective face number alone drove performance; as did subjective face number; but in this case the interaction did not so strongly modulate either main effect because of the reduced number of objective face conditions (only 1 or 3 ).

This provides evidence that not only the objective number of faces shown, but also the subjective number of faces reported, drives behavioral performance. Importantly, the effect of subjectively-reported number of perceived faces was also found in the CDA data, where we observed a main effect of number of subjective faces $(\beta=-0.09, F(1,4962)=6.25, p=0.012)$, no main effect of the objective number of faces $(\beta=-0.04, F(1,4962)=1.45, p=0.23)$, and an interaction between these two factors $(\beta=0.04, F(1,4962)=7.57, p=0.006)$, such that when more objective faces were shown, the subjective recognition of these faces was critical to performance rather than their mere presence.

When splitting the data into trials where participants reported seeing 0 or 1 faces and trials where participants reported seeing 2 or 3 faces (only using trials in which we showed 3 ambiguous faces), we found that performance was higher on trials where participants reported seeing more faces vs. less faces $(t(18)=2.83, p=0.011$; see Figure 5A). In addition, the CDA amplitude was larger for trials where more faces were recognized during encoding compared to trials where fewer faces were seen $(t(13)=2.35, p=0.035$; Fig. $4 \mathrm{C} \& 5 \mathrm{~B})$.

Together, these data show that active storage in visual working memory is modulated by meaningfully recognized stimuli, with such stimuli recruiting additional storage capacity. 
A

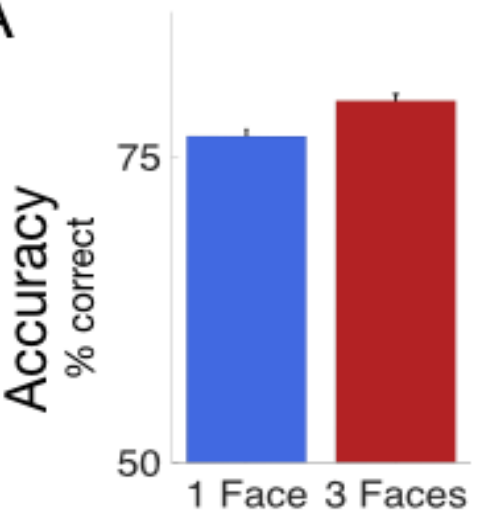

Number of faces shown

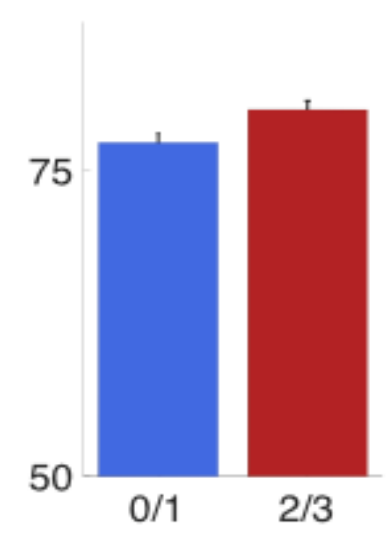

Number of faces reported when three were shown

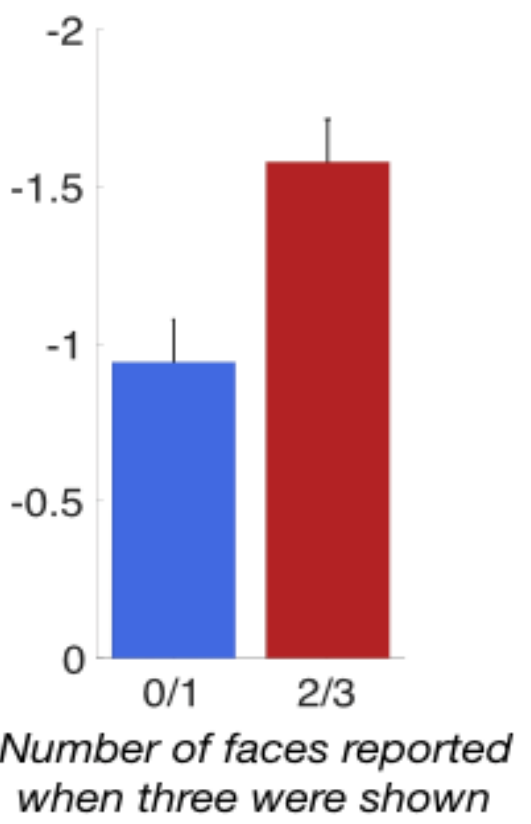

Figure 5. Results of Experiment 3 (A) Accuracy (\% correct) was higher when the memory display consisted of 3 faces vs. 1 face (left), and when participants reported seeing 2 or 3 faces vs. 0 or 1 faces on three-faces trials (right). (B) Mean CDA amplitude was higher when the memory display consisted of 3 faces $v$ s. 1 face (left), and when participants reported seeing 2 or 3 faces vs. 0 or 1 faces (right).

\section{General Discussion}




\section{EFFECT OF STIMULUS MEANING ON VWM}

The present results suggest that visual working memory performance is heavily influenced by the type of information being stored. Specifically, we found that an observer's ability to extract meaningful information from a stimulus enables them to hold more information actively in visual working memory, even for physically identical stimuli. Thus, our data suggest that visual working memory capacity is not fixed to a certain number of objects or a fixed pool of resources based on basic visual features (e.g., Awh, Barton \& Vogel, 2007; Luck \& Vogel, 2013; Bays et al. 2009), but critically depends on the type of information that is remembered - not how visually complex that information is, but rather whether that information can be perceived as a meaningful unit or not. In addition, our data argue against theories of visual working memory that propose that when having to remember the visual details of an object, as in the current task, persistence of sensory-like activity in low-level visual regions alone is responsible for memory performance (e.g., Serences, 2016); instead arguing for a major role for high-level interpretations of these visual details.

\section{Active storage measured via the CDA}

Active storage of information, and thus the use of working memory, is reflected in persistent neural activity in frontal and parietal brain regions (Buschman et al., 2011), and can be measured in the electroencephalogram with the CDA. The CDA is often taken as an index of the focus of attention in visual working memory -- e.g., the active storage component of the memory system. In particular, evidence for its selectivity to active storage in visual working memory includes: (1) Its magnitude increases with the number of items participants hold in working memory (Vogel \& Machizawa, 2004) and decreases when items are dropped from working memory (Vogel et al., 2005; Williams \& Woodman, 2012); (2) the CDA correlates with individual's working memory capacity (Vogel \& Machizawa, 2004; Störmer, Li, Heekeren, \& Lindenberger, 2013); (3) and most importantly, the CDA disappears when items have been consolidated into episodic longterm memory (Carlisle, Arita, Pardo, \& Woodman, 2011; Schurgin et al. 2018). Thus, the fact that we found an increased CDA for recognizable vs unrecognizable images demonstrates that the capacity increase observed behaviorally is mediated by greater ability to actively hold meaningful information in visual working memory rather than the use of other memory systems (like 'offline' storage in visual 'long'-term memory). Importantly, the CDA increase was present regardless of the visual complexity of the stimuli. This provides direct evidence that meaningfulness - rather than the visual information on our retinas - underlies visual working memory.

Our data are not consistent with an account where people have a fixed number of 'slots' or other memory resources and that unrecognizable shape stimuli simply take up more of these 'slots' or resources since they cannot be organized (as has sometimes been claimed about polygons and other complex stimuli; see Luria et al. 2010). This is because such an account predicts either equivalent CDA engagement in the two conditions, since in both cases working memory is 'full', or, if anything, greater CDA in the condition with the unrecognizable shapes, as they each require multiple slots or greater resource usage in memory. Instead, we find that the 


\section{EFFECT OF STIMULUS MEANING ON VWM}

face stimuli not only result in better performance but also greater sustained neural activity as measured by the CDA, suggesting the recruitment of additional memory resources for the meaningful stimuli. This is in direct contrast to the predictions of such fixed capacity accounts of visual working memory.

\section{Knowledge and visual working memory}

The observed benefits for working memory performance from recognizing faces and for remembering real-world objects (Brady et al. 2016) suggest that associations with meaningful conceptual information is the critical attribute that gives rise to enhanced active storage, similar to the role of meaning in long-term memory (Bower, Karlin, \& Dueck, 1975; Konkle et al., 2010; McWeeny, Young, Hay, \& Ellis, 1987; Brady et al. 2019). What determines the meaningfulness of an object? The present findings show that when a visual stimulus is associated with a high-level object category - for example a face, - this can be sufficient to increase working memory performance. This suggests that high-level visual templates (i.e., knowing how a face looks like) can provide one link to connect an incoming stimulus to existing knowledge and familiar categories. In this case meaningfulness may mostly depend on high-level visuallyderived categories, but in other cases, for example real-world objects, it seems plausible that other, even richer conceptual associations are being activated to induce meaningfulness. Such recognition may allow the engagement of more high-level brain regions, allowing people to maintain neural activity over the delay not only in low-level sensory information but also in high-level regions relevant to the processing of the stimulus (e.g., Druzgal \& D'Esposito, 2001; Stojanoski et al. 2019).

Many studies have shown that existing knowledge or familiarity with a stimulus improves the ability to maintain information not only in long-term memory, but also over short delays, often termed long-term working memory (Ericsson \& Kintsch, 1995). However, it has been unclear the extent to which such benefits arise from changes in active storage in working memory per se, or from the usage of 'long-term' memory systems or other forms of more offline storage that can be utilized in the short-term maintenance of information. Both neurally and cognitively, the active component of working memory (sometimes referred to as the 'focus of attention') is particularly important as it seems to be the element of working memory most responsible for capacity limits and correlations with broader cognitive abilities (e.g., Cowan, 2005). By contrast, other forms of memory sometimes called working memory but which are non-active -- like activated long-term memories or long-term working memory -- are widely acknowledged to be influenced by knowledge and meaningfulness (Ericsson \& Kintsch, 1995), but are often considered not to be core elements of working memory capacity (Awh \& Vogel, 2020). We here show that this active component of working memory (as indexed by the CDA) is strongly affected by the meaningfulness of the stimulus being remembered. If a stimulus connects to a concept and is thus perceived as meaningful, the active storage component of memory capacity is greater. 


\section{EFFECT OF STIMULUS MEANING ON VWM}

While a large body of work has shown benefits for objects of expertise in short-term storage (e.g., Curby, Glazek \& Gauthier, 2009), one line of recent work has claimed that expertise effects in visual working memory may sometimes arise from enhanced consolidation of items into memory rather than increased capacity per se (Xie \& Zhang, 2017b, 2018), although capacity increases have also been found (Xie \& Zhang, 2017a). Importantly, in the current work with our within-subject, perceptual manipulation of meaningful recognition and concurrent electrophysiological recordings, we show that active storage in working memory itself is impacted by meaning, with no change over the time course of the CDA component as suggested by a consolidation-based account. The kind of meaningfulness Xie and Zhang manipulate is quite different than in the current work, as unlike the recognition of a stimulus as a face, which recruits well-established regions of high-level cortex that could be used for enhanced storage, memory in their experiments likely requires retrieval of specific exemplar information, and it is unlikely participants have dedicated neural populations that can sustain activation for familiar but not unfamiliar Pokémon.

How might processing a stimulus as meaningful enhance active storage in working memory? One hypothesis is that active maintenance is limited by interference between the neural populations that must be held active (e.g., Cohen et al. 2014). Because having more relevant, meaningful information about an object or object category results in more relevant neural populations being activated, attentional mechanisms can maintain more active information successfully when dealing with meaningful objects (Wyble et al. 2016). Thus, knowledge may serve as a 'hook' that helps recruit the critical neural populations that are both more relevant and more distinct, thereby facilitating active maintenance of meaningful information. 


\section{Acknowledgements}

This work was supported by an NSF grant (BCS-1829434) to TFB and VSS.

\section{Author Contributions}

All authors contributed to the study concept and design. Data collection and analysis was conducted by IEA under supervision of VSS and TFB. IEA, VSS, and TFB wrote the manuscript and all authors approved the final version of the manuscript for submission.

\section{References}

Awh, E. \& Vogel, E.K, (2020) Online and Off-Line Memory States in the Human Brain. In the Cognitive Neurosciences, edited by Poeppel, Mangun \& Gazzaniga.

Awh, E., Barton, B., \& Vogel, E. K. (2007). Visual Working Memory Represents a Fixed Number of Items Regardless of Complexity. Psychological Science, 18(7), 622-628. https://doi.org/10.1111/j.1467-9280.2007.01949.x

Bartlett, F. C. (1932). Remembering: An experimental and social study. Cambridge: Cambridge University.

Bower, G. H., Karlin, M. B., \& Dueck, A. (1975). Comprehension and memory for pictures. Memory \& Cognition, 3(2), 216-220. https://doi.org/10.3758/BF03212900

Brady, T. F., Alvarez, G. A., \& Störmer, V. S. (2019). The role of meaning in visual memory: Face-selective brain activity predicts memory for ambiguous face stimuli. Journal of Neuroscience, 39(6), 1100-1108.

Brady, T. F., Störmer, V. S., \& Alvarez, G. A. (2016). Working memory is not fixed-capacity: More active storage capacity for real-world objects than for simple stimuli. Proceedings of the National Academy of Sciences, 113(27), 7459-7464. https://doi.org/10.1073/pnas.1520027113

Buschman, T. J., Siegel, M., Roy, J. E., \& Miller, E. K. (2011). Neural substrates of cognitive capacity limitations. Proceedings of the National Academy of Sciences of the United States of America, 108(27), 11252-11255.

Carlisle, N. B., Arita, J. T., Pardo, D., \& Woodman, G. F. (2011). Attentional Templates in Visual Working Memory. Journal of Neuroscience, 31(25), 9315-9322. https://doi.org/10.1523/JNEUROSCI.1097-11.2011

Cohen, M. A., Konkle, T., Rhee, J. Y., Nakayama, K., \& Alvarez, G. A. (2014). Processing multiple visual objects is limited by overlap in neural channels. Proceedings of the National Academy of Sciences, 111(24), 8955-8960.

Cowan N. Working memory capacity. Hove, East Sussex, UK: Psychology Press; 2005.

Curby, K. M., Glazek, K., \& Gauthier, I. (2009). A visual short-term memory advantage for objects of expertise. Journal of Experimental Psychology: Human Perception and Performance, 35(1), 94-107. https://doi.org/http://dx.doi.org/10.1037/0096-1523.35.1.94 


\section{EFFECT OF STIMULUS MEANING ON VWM}

Delorme, A., \& Makeig, S. (2004). EEGLAB: an open source toolbox for analysis of single-trial EEG dynamics including independent component analysis. Journal of neuroscience methods, 134(1), 9-21.

Druzgal, T. J., \& D'Esposito, M. (2001). Activity in fusiform face area modulated as a function of working memory load. Cognitive Brain Research, 10(3), 355-364.

Ericsson, K. A., \& Kintsch, W. (1995). Long-term working memory. Psychological Review, 102(2), 211-245. https://doi.org/http://dx.doi.org/10.1037/0033-295X.102.2.211

Konkle, T., Brady, T. F., Alvarez, G. A., \& Oliva, A. (2010). Conceptual distinctiveness supports detailed visual long-term memory for real-world objects. Journal of Experimental Psychology: General, 139(3), 558-578.

Li, X., Xiong, Z., Theeuwes, J., \& Wang, B. (2020). Visual memory benefits from prolonged encoding time regardless of stimulus type. Journal of Experimental psychology. Learning, Memory, and Cognition.

Lopez-Calderon, J., \& Luck, S. J. (2014). ERPLAB: an open-source toolbox for the analysis of event-related potentials. Frontiers in Human Neuroscience, 8.

Luck, S. J., \& Vogel, E. K. (1997). The capacity of visual working memory for features and conjunctions. Nature, 390(6657), 279.

Luck, S. J., \& Vogel, E. K. (2013). Visual working memory capacity: from psychophysics and neurobiology to individual differences. Trends in cognitive sciences, 17(8), 391-400.

Luria, R., Sessa, P., Gotler, A., Jolicœur, P., \& Dell'Acqua, R. (2010). Visual short-term memory capacity for simple and complex objects. Journal of cognitive neuroscience, 22(3), 496-512.

Luria, R., Balaban, H., Awh, E., \& Vogel, E. K. (2016). The contralateral delay activity as a neural measure of visual working memory. Neuroscience and Biobehavioral Reviews, 62, 100-108. https://doi.org/10.1016/j.neubiorev.2016.01.003

McCollough, A. W., Machizawa, M. G., \& Vogel, E. K. (2007). Electrophysiological Measures of Maintaining Representations in Visual Working Memory. Cortex, 43(1), 77-94. https://doi.org/10.1016/S0010-9452(08)70447-7

McWeeny, K. H., Young, A. W., Hay, D. C., \& Ellis, A. W. (1987). Putting names to faces. British Journal of Psychology, 78(2), 143-149. https://doi.org/10.1111/j.20448295.1987.tb02235.x

Mooney, C. M. (1957). Age in the development of closure ability in children. Canadian Journal of Psychology/Revue Canadienne de Psychologie, 11(4), 219-226. http://dx.doi.org/10.1037/h0083717

Quirk, C., Adam, K. C. S., \& Vogel, E. K. (2020). No Evidence for an Object Working Memory Capacity Benefit with Extended Viewing Time. PsyArix. https://doi.org/10.31219/osf.io/pzy5q

Schurgin, M. W., Cunningham, C. A., Egeth, H. E. and Brady, T.F. (2018). Visual Long-term Memory Can Replace Active Storage in Visual Working Memory. bioRxiv preprint. https://doi.org/10.1101/381848 


\section{EFFECT OF STIMULUS MEANING ON VWM}

Schwiedrzik, C. M., Melloni, L., \& Schurger, A. (2018). Mooney face stimuli for visual perception research. PLoS ONE, 13(7). https://doi.org/10.1371/journal.pone.0200106

Serences, J. T. (2016). Neural mechanisms of information storage in visual short-term memory. Vision Research, 128, 53-67. https://doi.org/10.1016/j.visres.2016.09.010

Stojanoski, B., Emrich, S. M., \& Cusack, R. (2019). Representation of semantic information in ventral areas during encoding is associated with improved visual short-term memory. bioRxiv.

Störmer, V. S., Alvarez, G. A., \& Cavanagh, P. (2014). Within-hemifield competition in early visual areas limits the ability to track multiple objects with attention. Journal of Neuroscience, 34(35), 11526-11533.

Störmer, V.S., Li, S.-C., Heekeren, H.R., Lindenberger, U. (2013). Normative shifts of cortical mechanisms of encoding contribute to adult age differences in visual-spatial working memory.NeuroImage, 73, 167-175.

Vogel, E. K., \& Machizawa, M. G. (2004). Neural activity predicts individual differences in visual working memory capacity. Nature, 428(6984), 748-751. https://doi.org/10.1038/nature02447

Vogel, E. K., McCollough, A. W., \& Machizawa, M. G. (2005). Neural measures reveal individual differences in controlling access to working memory. Nature, 438(7067), 500503. https://doi.org/10.1038/nature04171

Williams, M., \& Woodman, G. F. (2012). Directed forgetting and directed remembering in visual working memory. Journal of Experimental Psychology: Learning, Memory, and Cognition, 38(5), 1206-1220. https://doi.org/http://dx.doi.org/10.1037/a0027389

Wiseman, S., \& Neisser, U. (1974). Perceptual Organization as a Determinant of Visual Recognition Memory. The American Journal of Psychology, 87(4), 675-681. https://doi.org/10.2307/1421974

Wyble, B., Swan, G., \& Callahan-Flintoft, C. (2016). Measuring Visual Memory in Its Native Format. Trends in Cognitive Sciences, 20(11), 790-791

Xie, W. \& Zhang, W. (2017a). Familiarity increases the number of retained Pokémon in visual short-term memory. Memory \& Cognition. 45(4), 677-689

Xie, W. \& Zhang, W. (2017b). Familiarity speeds up visual short-term memory consolidation. Journal of Experimental Psychology-Human Perception and Performance. 43(6), 12071221.

Xie, W \& Zhang, W. (2018). Familiarity speeds up visual short-term memory consolidation: Electrophysiological evidence from Contralateral Delay Activities. Journal of Cognitive Neuroscience, 30(1), 1-13

Zhang, W., \& Luck, S. J. (2008). Discrete fixed-resolution representations in visual working memory. Nature, 453(7192), 233-235. https://doi.org/10.1038/nature06860 


\section{Supplemental information}

\section{Statistical Analysis Experiment 1}

Experiment 1 has a within-subject, 1-factor design with 4 levels (the number of stimuli that are upright faces, 0-3), as well as another factor, the subjectively reported number of faces seen (also $0-3)$. The dependent measure is accuracy in the 2-AFC memory test. As described in the preregistration, our main analysis uses a Generalized Linear Mixed Effect model, taking all of the trials accuracy data (0/1, binomial) and fitting a model that treats both participants and items as random effects. We ask - separately and together - about the effect of the objective number of faces shown $(0,1,2,3)$ and subjective number of reported faces $(0,1,2,3)$. In particular, to look at the effect of objective number of faces alone, we examine the effect of the objective number of faces shown after taking into account random effects of participants and items:

$$
\text { Correct } \sim 1+\text { ObjectiveFaceNum }+(1 \mid \text { Item })+(1 \mid \text { Subject })
$$

In a separate model, the effect of both objective and subjective number of faces and their interaction is estimated, again treating both subjects and items as random effects:

$$
\text { Correct } \sim 1+\text { ObjectiveFaceNum } * \text { SubjectiveFaceNum }+(1 \mid \text { Item })+(1 \mid \text { Subject })
$$

We fit these models using the fitglme function in MATLAB, with a binomial distribution. We obtained significance ( $\mathrm{p}$-values) using MATLAB's defaults for the anova function (a Type III Ftest). These models allow us to examine how accuracy is impacted by both the objective and subjective number of faces present, while taking into account item effects.

\section{EEG Experiment 3 Methods}

Each trial started with the presentation of six dots that served as placeholders; three were positioned on the left half of the screen in a semicircle and the other three were presented on the right half of the screen. These dots appeared for 500-800ms (jittered) and served as location markers for the later images. Then six images appeared on the screen as well as a central arrow cue; three images were positioned on the left half of the screen and the other three were presented on the right half of the screen. The central arrow cue, indicating which side to memorize, appeared at the same time as the stimuli. The images were presented for $1200 \mathrm{~ms}$ to allow ample time to recognize the arrow cue, shift attention to the cued side covertly, and encode the images. Participants were required to maintain fixation while the images were on the screen as well as during the delay period. Therefore, participants could only use their peripheral vision to study the images on the cued side, and compliance with this was ensured by rejecting trials with horizontal eye motion from the analysis (see Electrophysiological Recordings and Analysis below). After the images disappeared, there was an $800 \mathrm{~ms}$ delay period with only location 


\section{EFFECT OF STIMULUS MEANING ON VWM}

markers on the screen. Following the delay, one of the location markers on the cued side was enlarged and presented for $500 \mathrm{~ms}$, indicating the location of the to-be-tested image. Once the test images appeared, participants were allowed to blink and move their eyes. The experiment consisted of 10 blocks with 32 trials in each. The trial conditions ( 1 vs. 3 faces in each study array) were randomized throughout the experiment and each trial condition was presented evenly within each block. Prior to the experiment, all participants completed two mini-blocks of 4 trials each as practice for the full 10 experimental blocks that followed. None of the images used in the practice trials were used in the full experiment.

\section{EEG recordings and EEG analysis Experiment 3}

EEG activity was recorded continuously from $32 \mathrm{Ag} / \mathrm{AgCI}$ electrodes arranged according to the 10-20 system, mounted in an elastic cap and amplified by an ActiCHamp amplifier (Brain Products $\mathrm{GmbH}$ ). The horizontal electro-oculogram was acquired using a bipolar pair of electrodes positioned at the external ocular canthi, and the vertical electro-oculogram was measured at electrode FP1, located above the left eye. All scalp electrodes were referenced to an electrode on the right mastoid online, were digitized at a rate of $500 \mathrm{~Hz}$, and a low-pass filter of $1000 \mathrm{~Hz}$ was applied during recording. Signal processing was performed with MATLAB (The MathWorks) using the EEGLAB (Delorme \& Makeig, 2004) and ERPLAB toolboxes (LopezCalderon \& Luck, 2014) and custom-written scripts. Continuous EEG data was filtered offline with a bandpass of $0.01-112 \mathrm{~Hz}$ (half amplitude cut-off, with a slope of $-12 \mathrm{~dB} /$ octave). Data were epoched into trials and artifact rejection was performed for the interval -200 to $+2000 \mathrm{~ms}$ relative to the memory display onset and trials with horizontal eye movements, blinks, or excessive muscle movements were excluded from the analysis.

Artifacts were detected using a semiautomated procedure, in which differences between minimum and maximum voltages were compared with a threshold value. Threshold values were determined by visual inspection for each subject individually. After using the artifact detection functions implemented in ERPLAB (Lopez-Calderon \& Luck, 2014; peak-to-peak for blinks at channel FP1, and a step function to detect horizontal eye movements at the HEOG channel), we re-checked the artifact detection visually to ensure that all eye movements and blinks were detected correctly. If additional artifacts were spotted, these trials were excluded as well. For the subjects retained in the final data set $(\mathrm{N}=19)$, this resulted in an average rejection rate of $18.3 \%$.

\section{HEOG Analysis:}

To ensure that any lateralized effects indexed by the CDA component were not due to differences in spurious eye movements between the conditions, we compared the HEOG waveforms for remember-left vs. remember-right conditions during the CDA time window separately for the two set sizes (1-face vs. 3-faces), and found no reliable difference for each of the left-vs.-right comparisons $(t(18)=1.64 ; p=0.12$ for the 1 -face condition, and $t(18)=1.38$; $p=0.19$ for the 3 -face condition). Most importantly, the left-minus-right difference waves did not 
EFFECT OF STIMULUS MEANING ON VWM

differ between the conditions $(t(18)=0.16 ; p=0.87$; see Supplementary Figure 1$)$, demonstrating that the CDA differences we observed between conditions are not due to differences in eye movements.

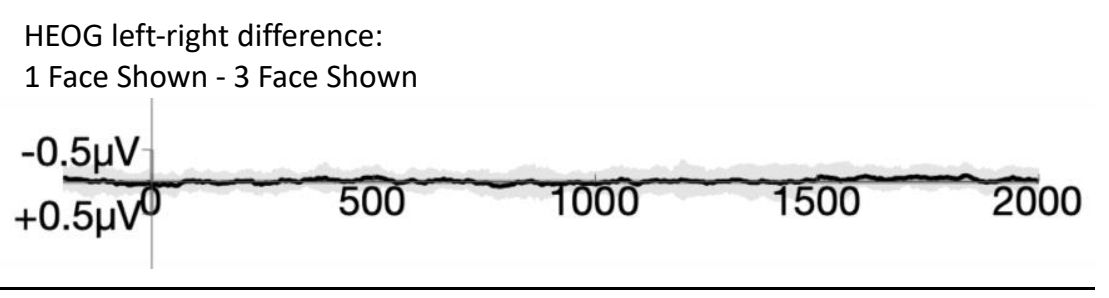

Supplementary Figure 1: We found no eye drifts that could affect the CDA magnitude differentially: As reported in the Methods, we found no significant HEOG difference for remember-left vs. remember-right trials in either condition separately. In addition, plotted here is the difference-of-differences: If small differences in left-right eye drift caused the difference in CDA between 1 face shown vs 3 face shown, this would be reflected in a deviation from 0 in this plot, which is not present.

\section{Statistical Analysis Experiment 3:}

Experiment 3 has a within-subject, 1-factor design with 2 levels (the number of stimuli that are faces, 1 or 3), and another factor of the subjectively reported number of faces (0-3).

The behavioral dependent measure is again accuracy in the 2-AFC memory test. In addition to fitting the same Generalized Linear Mixed Effect model from Experiment 1 and 2,the effect of objective number of faces can now also be compared via a paired t-test since there are only 2 levels.

For comparing CDA based on the objective and subjective number of faces, we also rely on a Linear Mixed Effect model to take into account not only random effects of subjects but also items, taking advantage of the benefits of a mixed-effects analysis for electrophysiology (Bagiella, Sloan, \& Heitjan, 2000). In particular, we calculate CDA on a per trial basis by: (1) first subtracting the baseline separately for each relevant electrode for each trial, (2) then subtracting contralateral electrodes from ipsilateral electrodes; and then (3) averaging over the CDA time window. Then we fit a model that takes into account either only the objective number of faces:

$$
\text { CDA } ~ 1+\text { ObjectiveFaceNum }+(1 \mid \text { Item })+(1 \mid \text { Subject })
$$

or both the objective and subjectively reported number of faces:

$$
\text { CDA } ~ 1+\text { ObjectiveFaceNum*SubjectiveFaceNum + (1|Item })+(1 \mid \text { Subject })
$$

We fit these models using the fitlme function in MATLAB, using a rank-based inverse normal transformation (INT) on CDA values (Bliss, 1967). Nearly identical results were obtained when 


\section{EFFECT OF STIMULUS MEANING ON VWM}

using untransformed CDA values, but the residuals were not normally distributed, so the transformation was necessary to meet the assumptions of the statistical test. We obtained significance (p-values) using MATLAB's defaults for the anova function (a Type III F-test). In addition, to test the effect solely from the objective number of faces without controlling for item effects, we report a paired t-test of CDA magnitude.

\section{Supplementary Table 1}

The table reports the frequency with which trials were present in these bins, for both this experiment and Exp. 1 and 2. As expected, and consistent with this analysis, participants do not report seeing the exact number of faces that were being presented to them, allowing us to examine both the subjective perception of the stimuli as well as the objective number of faces presented.

\begin{tabular}{|l|l|l|l|l|}
\hline Experiment 1 & Reported 0 & Reported 1 & Reported 2 & Reported 3 \\
\hline Shown 0 & $51 \%$ & $32 \%$ & $16 \%$ & $2 \%$ \\
\hline Shown 1 & $22 \%$ & $52 \%$ & $24 \%$ & $3 \%$ \\
\hline Shown 2 & $11 \%$ & $31 \%$ & $48 \%$ & $10 \%$ \\
\hline Shown 3 & $6 \%$ & $19 \%$ & $37 \%$ & $39 \%$ \\
\hline Experiment 2 & Reported 0 & Reported 1 & Reported 2 & Reported 3 \\
\hline Shown 0 & $24 \%$ & $30 \%$ & $25 \%$ & $12 \%$ \\
\hline Shown 1 & $14 \%$ & $31 \%$ & $41 \%$ & $14 \%$ \\
\hline Shown 2 & $9 \%$ & $25 \%$ & $45 \%$ & $22 \%$ \\
\hline Shown 3 & $6 \%$ & $15 \%$ & $40 \%$ & $39 \%$ \\
\hline Experiment 3 & Reported 0 & Reported 1 & Reported 2 & Reported 3 \\
\hline Shown 1 & $21 \%$ & $38 \%$ & $31 \%$ & $10 \%$ \\
\hline Shown 3 & $5 \%$ & $25 \%$ & $42 \%$ & $28 \%$ \\
\hline
\end{tabular}

Table 1. Frequency of total trials reporting each subjective number of faces for each objective number of faces for each of the 3 experiments. 
EFFECT OF STIMULUS MEANING ON VWM 